American J. of Engineering and Applied Sciences 4 (1): 70-76, 2011

ISSN 1941-7020

(C) 2010 Science Publications

\title{
Applications of Conditional Value at Risk to Full Truckload Pricing Under Demand and Service Time Uncertainties
}

\author{
Thitima Wonginta and Sompong Sirisoponsilp \\ Department of Civil Engineering, Faculty of Engineering \\ Chulalongkorn University, Phayathai Road, Pathumwan, Bangkok 10330 Thailand
}

\begin{abstract}
Problem statement: Pricing is one of the fundamental management decisions required by a truckload carrier. Traditional pricing based on an average all relevant costs including fixed and variable costs is not capable of providing adequate margins that prevent losses during operation uncertainties inherent in truckload operation including demand variability and variation in service times. Approach: This study utilizes Conditional Value at Risk (CVaR) as a measure of risk with significant advantage over Value at Risk (VaR), to full truckload pricing when conditions are unpredictable. It criterion focuses on the tail of the loss distribution and provides a measure of the expected loss exceeding Value-at-Risk. Therefore, it was applied to control the maximum loss or the minimum gain within a specified tolerance level to enable more flexible full truckload pricing. A simulation model is developed to capture the stochastic patterns inherent in the operation of full truckload network. Results: Price per trip from $95 \% \mathrm{CVaR}$ is less than traditional pricing for delivery over short distances while extremely higher for delivery over long distances. We apply traditional prices back to the truckload operation and network imitated in the simulation model and find that even the traditional prices are set to include a certain percentage of profit over the average cost there is still a large chance that the carrier will be subjected to a loss. Conclusion: The numerical analysis for this study demonstrates a pricing method for transportation carriers who are risk averse. Transportation carriers in this group dislike risk and will stay away from high risk.
\end{abstract}

Key words: Conditional Value at Risk (CVaR), full truckload pricing, risk measurement, Less-ThanTruckload (LTL), potential customers, proposed methodology, simulation model, numerical analysis, fundamental management

\section{INTRODUCTION}

Today truck transportation is the dominant mode of freight transportation in Thailand. Several studies have revealed that over $80 \%$ of domestic freight movement by weight is currently served by trucks and that truck transportation demand continues to rise dramatically in conjunction with the nation's high economic growth. Truckload service plays a major role in Thailand because a large proportion of freight moved in Thailand lends itself to truckload movements, such as bulk agriculture products and construction materials. In Thailand, the truckload carrier market is highly competitive due to the ease of market entry resulting from the intrinsic simplicity of Truckload operation which provides point-to-point trucking services compared to Less-Than-Truckload (LTL) operation which requires a network of local terminals for consolidation and break-bulking activities.

Given the extremely competitive nature of the market, pricing is a key driver of business success.
Specifying the "right" price offering to potential customers is a challenging task which can affect the long-term survival of the carriers, as over-pricing will turn potential customers away while under-pricing will result in eventual financial losses. Moreover, pricing trucking services is certainly a difficult task if one considers the various uncertainties that may possibly affect the complexities and the cost of trucking operation. These uncertainties include not only those internal to the carrier operation such as the availability of trucks, but also those that lie outside the direct control of carriers such as gasoline price, customer demand and road accidents.

Among all external uncertainties encountered in daily truckload operation, variability in demand is possibly the most important factor because it can simultaneously affect both the revenue and the cost of a trucking operation. Higher-than-expected demand may be favorable in the first instance because it means greater revenue but the unexpected demand will have severely

Corresponding Author: Thitima Wonginta, Department of Civil Engineering, Faculty of Engineering,

Chulalongkorn University Phayathai Road, Bangkok 10330 Thailand 
adverse effects on the operation and the additional cost of mobilizing resources to serve this unforeseen demand may be greater than the revenue earned.

The second most significant source of uncertainties in truckload operation is the time required to complete the delivery, because this will affect the use of available trucks. As truckload movements usually involve intercity long-haul movement, the transit time is relatively constant, but the time associated with the waiting at the customers' premises and the loading/unloading of vehicles may vary greatly among different shipments due to changing customer requirements. Customer demand and service time are the two factors of uncertainties which will be considered in this study.

Uncertainty gives rise to risk, the potential of loss (Suri and Soni, 2006). The literature describes various risk various risk measures which can be used to evaluate a system's riskiness. Over the past few years, the financial engineering field's financial managers have increasingly used Value at Risk (VaR). VaR is defined as the expected loss arising from an adverse market movement with specified probability over a period of time (Tapiero, 2005; Saleh et al., 2009). It answers the question of how much one can lose with $\mathrm{x} \%$ probability over a period of time. It suffers; however, from being unstable and difficult to work with numerically when losses are not "normally" distributed. In fact, it is often the case because loss distributions tend to exhibit "fat tails". A very serious shortcoming of $\mathrm{VaR}$, in addition, is that it provides no handle on the extent of losses that might be suffered beyond the amount indicated by this measure. An alternative measure that does quantify the losses that might be encountered in the tail of the loss distribution is conditional value at risk, or CVaR. It was first introduced by Rockafellar and Uryasev (2000), Uryasev (2000) as an extension to Value at Risk. Hence, in order to control the risk of highest loss, we apply a $\mathrm{CVaR}$ constraint to estimate full truckload pricing in this article. Full truckload pricing is considered with the probability of an acceptable loss which is less than the expected target under a specified confidence level of $\mathrm{CVaR}$. Although CVaR has not become a standard in the finance industry, CVaR is likely to play a major role as it currently does in insurance industry (Embrechts et al., 1997). CVaR has been proofed as a more consistent measure of risk since it is sub-sdditive and convex (Artzner et al., 1999). The CVaR concept is particularly relevant for the truckload industry because as the market is extremely competitive the carriers are pricetakers rather than price-setters.

By definition with respect to a specified probability level $\alpha$, the $\alpha-\mathrm{V} \alpha \mathrm{R}$ of a portfolio is the lowest amount $\beta$ such that, with probability $\alpha$, the loss will not exceed $\beta$, whereas the $\alpha-C V \alpha R$ is the conditional expectation of losses above that amount $\beta$. CVaR measures the conditional expected loss exceeding VaR and accounts for the risks beyond the $\mathrm{VaR}$ value. The $\mathrm{CVaR}$ measure is able to quantify dangers beyond the VaR value. To avoid the undesirable characteristics of VaR, Conditional Value-at-Risk (CVaR) will be applied as an alternative measure of risk, with more attractive properties.

The $\alpha-C V \alpha R$ values for the loss random variable associated with xand any specified probability level $\alpha$ will be denoted by $\phi \alpha(\mathrm{x})$, which are given by:

$$
\varphi_{\alpha}(\mathrm{x})=(1-\alpha)^{-1} \int_{\mathrm{f}(\mathrm{x}, \mathrm{y}) \beta_{\alpha}(\mathrm{x})} \mathrm{f}(\mathrm{x}, \mathrm{y}) \mathrm{p}(\mathrm{y}) \mathrm{dy}
$$

In the Eq. 1 , the probability that $f(x, y) \geq \beta_{\alpha}(x c)$ is therefore equal to $1-\alpha$. Therefore, $\phi \alpha(\mathrm{x})$ comes out as the conditional expectation of the loss associated with $\mathrm{x}$ relative to that loss being $\beta_{\alpha}(\mathrm{x})$ or greater. It can be ensured that the $\alpha-V \alpha R$ is never more than the $\alpha-C V \alpha R$, that means $\mathrm{CVaR}$ will naturally give low $\mathrm{VaR}$ as well.

\section{MATERIALS AND METHODS}

The objective of this research is to develop a methodology for determining reasonable service prices to offer to a new customer. The proposed methodology has two basic components, namely the full truckload simulation model and the full truckload pricing model, as illustrated in Fig. 1.

Full truckload simulation model: When a new customer contacts a truckload carrier for service, the customer will have a relatively firm idea on the total volume of freight to be served but will not know exactly how the demand will vary from day-to-day. Moreover, the times required for a truck to wait at the customer site and to complete loading/unloading may experience daily fluctuation. The truckload simulation model is developed in a spreadsheet program using Visual Basic to capture these uncertainties in demand and service times. With the assumption that the carrier presently provides service to certain prior customers, the simulation model can be used to analyze the impacts of new customers' service requests on the daily operation rendered to both new and existing customers. The simulation outputs show daily operating costs and provide a number of performance measures.

In the developed simulation model the carrier has a fixed truck fleet and current customers will have priority over new customers. In other words, available trucks will be first assigned to serve existing customers' 


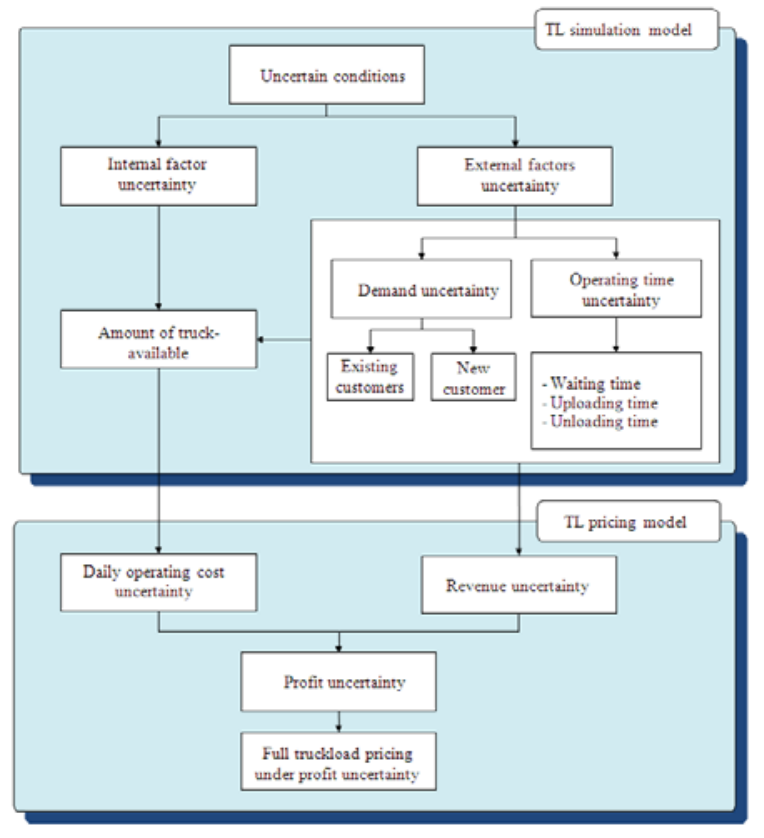

Fig. 1: Research framework

demand and the remaining trucks in the fleet will then be assigned to serve the new customer in everyday operation. If there are not enough trucks, the carrier will have to request additional trucks from other subcontract companies at a relatively high cost. In serving a shipment the designated truck will process through the following stages: moving to the shipment origin, waiting for loading, loading, moving to the destination, waiting for unloading, unloading, moving on to the next assignment (if any).

Full truckload pricing model: A full truckload pricing model is developed by applying the Value at Risk (VaR) risk optimization technique to determine the minimum service price offering by controlling the risk of earning less than the desired profit or losing more than an acceptable level due to uncertain factors. The profit model is simply equal to revenue earned that depends on demand minus a set operating cost and a total fixed cost. Let $\pi(p, z)$ be the profit associated with the decision vector $p$, which represent transportation pricing in term of baht $/ \mathrm{km}$ and the random vector $\mathrm{z}$, which including two factors that are demand and operating time uncertainty. Thus for each $\mathrm{p}$, the profit $\pi(\mathrm{p}, \mathrm{z})$ is a random variable having a distribution induced by $\mathrm{z}$. Assume the underlying probability density function of random variables is denoted by $\operatorname{Pr}(\pi(\mathrm{p}, \mathrm{z}))$. For the $\mathrm{VaR}$ profit is the value of the $\alpha$-quantile of the total profit, e.g., at $95 \%$ confident level or a $5 \%$ chance that earnings would yield a less than $\mathrm{VaR}$. CVaR is the average of $5 \%$ the tail of profit distribution.

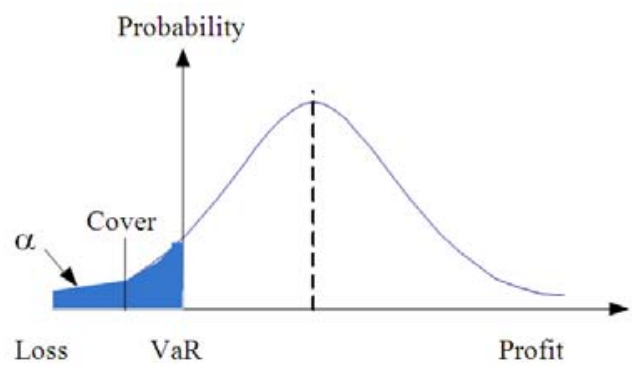

Fig. 2: Probability of loss area

$\mathrm{CVaR}$ is seen to be more sensitive to samples with very long tail distribution loss from high operating cost compare to VaR. However, CVaR is more stable with changes in the $\alpha$-value. This can be explained by the fact that CVaR is an average of the tail, whereas $\mathrm{VaR}$ is the quantile defining the tail. The quantile is likely to jump with a small sample, whereas the average will shrink this effect (Dehlendorff et al., 2010).

In this article, to minimize service prices for new customers we apply Conditional Value at Risk ( $\mathrm{CVaR}$ ) as a constraint at the value of $\alpha$-quantile as illustrated in Fig. 2.

Then, the optimization problem can be described as follows.

\section{Objective min p subject to:}

$\mathrm{CVaR}_{\mathrm{a}}\left[\operatorname{Pr}(\pi(\mathrm{p}, \mathrm{z}) \geq] \pi_{0} \mathrm{p} \geq 0\right.$

List of notations:

$\pi(\mathrm{p}, \mathrm{z})=$ Profit function with demand and operating time uncertainty

$\pi_{0} \quad=$ Minimum acceptable gain $\left(\pi_{0}=0\right)$

$\alpha=$ Threshold probability value of the downside risk constraint

$\mathrm{P} \quad=$ Full truckload price per distance

\section{RESULTS}

Input Data: To illustrate how full truckload pricing operates, let us now consider a numerical example using a simulated dataset generated from historical data provided by a truckload carrier located in Bangkok, Thailand. As illustrated in Fig. 3, the first step generates the trucking demand requested by current and new customers and assumes that the demands follow normal distribution. In the model a single type of trucks (six-wheeled trucks) is used. The average daily demands are specified as 10 and 9 shipments day $^{-1}$ for current and new customers respectively. 
Am. J. Engg. \& Applied Sci., 4 (1): 70-76, 2011

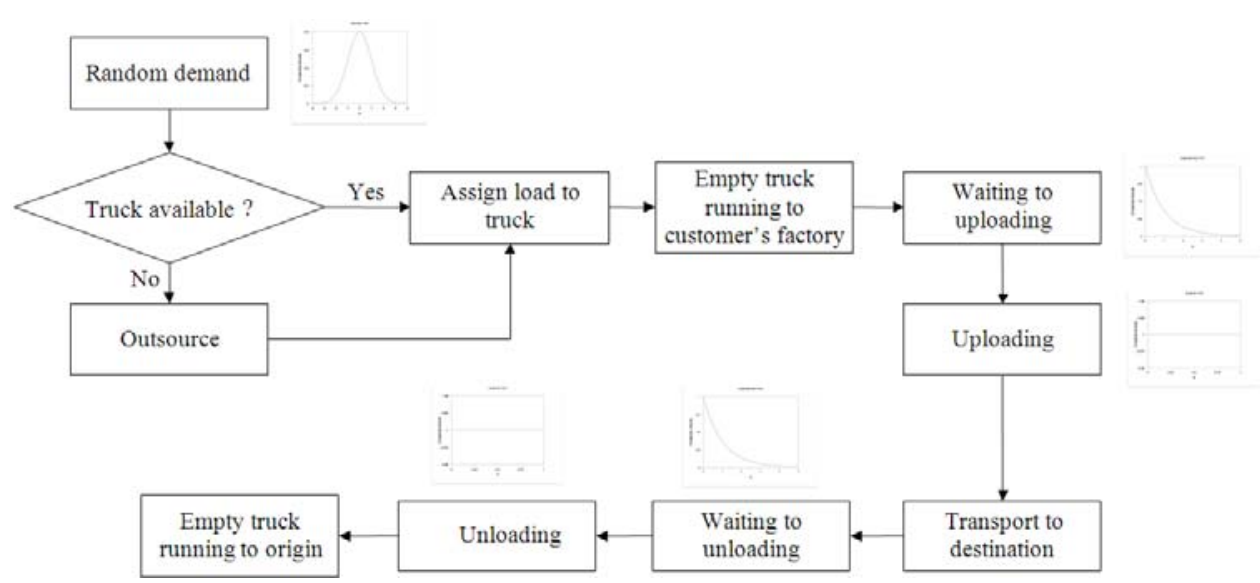

Fig. 3: Process flow for full truckload simulation

Table 1: Customer's demand information

\begin{tabular}{|c|c|c|c|c|c|}
\hline & Destination & Distance (km.) & $\begin{array}{l}\text { Average demand } \\
\text { (shipments day }^{-1} \text { ) }\end{array}$ & $\begin{array}{l}\text { Std. }(10 \% \text { of } \\
\left.\text { Demand day }{ }^{-1}\right)\end{array}$ & $\begin{array}{l}\text { Outsourcing cost } \\
\text { (baht/trip) }\end{array}$ \\
\hline \multirow[t]{4}{*}{ Current customer } & Nakhon Ratchasima & 259 & 3 & 0.3 & 7.562 \\
\hline & Khon Kaen & 449 & 2 & 0.2 & 13.753 \\
\hline & Surat Thani & 644 & 2 & 0.2 & 18.835 \\
\hline & Phitsanuklok & 377 & 3 & 0.3 & 12.322 \\
\hline \multirow[t]{5}{*}{ New customer } & Phra Nakhon Si Ayutthaya & 76 & 3 & 0.3 & 4.323 \\
\hline & Chonburi & 81 & 2 & 0.2 & 4.621 \\
\hline & Udon Thani & 564 & 2 & 0.2 & 16.038 \\
\hline & Songkhla & 950 & 2 & 0.2 & 26.123 \\
\hline & Chiang Mai & 696 & 2 & 0.2 & 19.868 \\
\hline
\end{tabular}

Table 2: The proportion of extra trucks needed to meet average demand day ${ }^{-1}$

\begin{tabular}{lr}
\hline Total trucks & Outsourced Trucks day $^{-1}(\%)$ \\
\hline 22 & 46 \\
25 & 36 \\
30 & 24 \\
35 & 15 \\
40 & 8 \\
\hline
\end{tabular}

The destination probability or the probability that the shipment will be directed to a particular destination is specified based on historical data as illustrated in Table 1 . In addition, we assume that the uploading and unloading are uniformly distributed while waiting times for uploading and unloading are exponentially distributed. The average line-haul speeds of laden and empty trucks are specified as 50 and $70 \mathrm{~km} \mathrm{~h}^{-1}$ respectively.

Data Analysis: With all necessary parameters specified, we apply the developed simulation model to analyze the resulting operation performance and costs. To serve current customers demand, the carrier is assumed to operate 22 six-wheeled trucks. We run 100 simulations to imitate the real-life operation of 90 working days, using the existing number of trucks (22 six-wheeled trucks). This reveals that the existing number of trucks is not enough to serve both current and new customer demand. Simulation output shows that carriers need to outsource trucks from sub-contractors to meet about $46 \%$ of total daily demand. Requesting additional trucks from sub-contract companies is relatively expensive, so instead we increase the number of trucks and run the simulation again. The output performances of imitating real-life operation for 180 working days are illustrated in Table 2.

In this case, the carrier is assumed to desire less than $10 \%$ of average daily demand to be outsourced. This requires investing in 18 additional trucks to adequately serve the demand of new customers. When total customer demand exceeds the capacity of these trucks, outsourcing will result in added expense.

Using these adjusted figures, another simulation of real-life operation over 180 working days is run. Specific performance measures derived by the simulation model and used as inputs to the pricing model include daily transportation cost, outsourcing cost and fixed cost. Requests for outsourced trucks in the simulation could fall into two scenarios of truck assignment: (1) our own trucks are given the first priority for short-distance deliveries while outsourced trucks are reserved for long distances and (2) our own trucks are given first priority for long-distance deliveries while outsourced trucks are reserved for short distances. 
Am. J. Engg. \& Applied Sci., 4 (1): 70-76, 2011

Table 3: Comparing full truckload pricing applying with 95\% CVaR with traditional pricing per trip for the first scenario of truck assignment

\begin{tabular}{|c|c|c|c|c|c|}
\hline \multirow[b]{2}{*}{ Destination } & \multirow{2}{*}{$\begin{array}{l}\text { Average cost } \\
\text { (baht/trip) }\end{array}$} & \multicolumn{3}{|c|}{ Traditional pricing (baht/trip) } & \multirow{2}{*}{$\begin{array}{l}\text { Price with 95\% } \\
\text { CVaR (baht/trip) }\end{array}$} \\
\hline & & 5\% Profit & $10 \%$ Profit & 15\% Profit & \\
\hline Phra Nakhon Si Ayutthaya & 2.128 & 2.234 & 2.340 & 2.447 & 1.566 \\
\hline Chonburi & 2.193 & 2.302 & 2.412 & 2.522 & 1.669 \\
\hline Udon Thani & 9.613 & 10.093 & 10.574 & 11.055 & 11.624 \\
\hline Songkhla & 15.771 & 16.560 & 17.349 & 18.137 & 19.580 \\
\hline Chiang Mai & 11.899 & 12.494 & 13.089 & 13.684 & 14.345 \\
\hline Probability of experiencing a loss & $100 \%$ & $100 \%$ & $9.50 \%$ & $5.00 \%$ & \\
\hline
\end{tabular}

Table 4: Comparing full truckload pricing applying with 95\% CVaR with traditional pricing per trip for the second case of truck assignment

\begin{tabular}{|c|c|c|c|c|c|}
\hline \multirow[b]{2}{*}{ Destination } & \multicolumn{4}{|c|}{ Traditional pricing (baht/trip) } & \multirow{2}{*}{$\begin{array}{l}\text { CVaR (baht/trip) } \\
\text { Price with 95\% }\end{array}$} \\
\hline & 5\% Profit & 7\% Profit & 10\% Profit & 15\% Profit & \\
\hline 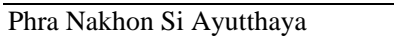 & 2.234 & 2.277 & 2.340 & 2.447 & 1.463 \\
\hline Chonburi & 2.302 & 2.346 & 2.412 & 2.522 & 1.559 \\
\hline Udon Thani & 10.093 & 10.286 & 10.574 & 11.055 & 10.855 \\
\hline Songkhla & 16.560 & 16.875 & 17.349 & 18.137 & 18.285 \\
\hline Chiang Mai & 12.494 & 12.732 & 13.089 & 13.684 & 13.396 \\
\hline Probability of experiencing a loss & $94.40 \%$ & $31.80 \%$ & - & - & $5.00 \%$ \\
\hline
\end{tabular}

After running the simulation model, the pricing model was applied with 40 six-wheeled trucks to determine the minimum price offerings that would allow a downside risk constraint at $95 \%$ confident level or only a $5 \%$ chance that earnings would yield a less than acceptable loss.

The outputs from the pricing model in the first scenario of truck assignment reveal that the minimum pricing is 20.61 baht $\mathrm{km}^{-1}$. We then converted pricing per unit into full truckload pricing per trip. We compared pricing by controlling risk to achieve a loss with a traditional pricing method that is estimated by using cost-plus pricing method or estimated pricing from average cost plus percent of profit required. A comparison of the results is displayed in Table 3.

Price per trip from $95 \% \mathrm{CVaR}$ is less than traditional pricing for delivery over short distances while extremely higher for delivery over long distances. The most important reason is that the short-distance routes are mostly served by existing trucks left from serving current customers so that the depreciated cost is already allocated to current customers. Also, comparing additional depreciation from new truck owned by the company is still less than outsourcing cost. Meanwhile, serving some long distance routes with outsourced trucks is highly expensive. Hence the variable costs for short distance routes are lower and pricing will consequently be lower.

We apply these traditional prices back to the truckload operation and network imitated in the simulation model and find that even the traditional prices are set to include a certain percentage of profit over the average cost there is still a large chance that the carrier will be subjected to a loss. For example, although the carrier adds up as high as $15 \%$ of profit to the average cost, he will still be facing a $9.50 \%$ probability of incurring a loss.

In the second scenario of truck assignment, own trucks are reserved for long-distance trips. As illustrated in Table 4, the minimum pricing in this case is 19.25 baht $\mathrm{km}^{-1}$, which is less than pricing in the first case and the probabilities of loss are lower than those in the first scenario. It can be implied that the truck assignment rule highly affects cost and price.

Besides the truck assignment rule, other factors may strongly affect transportation cost and price. In this case, the simulation model can be further applied to consider the influence of the number of additional trucks to be purchased to serve a new customer. Applying the first truck assignment rule, we test the scenario by changing the number of additional trucks in the simulation model. The outputs indicate that at the beginning increasing the number of trucks leads to a lower cost and price. This logically follows from the fact that having more trucks of its own means that a company needs to outsource fewer trucks. However, we cannot increase the quantity of trucks infinitely each additional truck requires additional investment and a higher fixed cost. In this case, we can increase the size of the fleet by additional 24-25 sixwheeled trucks. After that it will generate higher cost and price as displayed in Fig. 4.

This numerical analysis demonstrates a pricing method for transportation carriers who are risk averse. Transportation carriers in this group dislike risk and will stay away from high risk. Hence, pricing with $95 \%$ of CVaR is agreeable to this kind of person. However, if they stay extremely risk averse, pricing will be very high as a result. 
Am. J. Engg. \& Applied Sci., 4 (1): 70-76, 2011

Table 5: Comparing full truckload pricing on different levels of risk by changing different confident level for the second case of truck assignment Price with different confident level of CVaR (baht km${ }^{-1}$ )

\begin{tabular}{llll} 
Additional number of trucks & $80 \%$ CVaR & $85 \%$ CVaR & $90 \%$ CVaR \\
\hline 18 & 19.10 & 19.14 & 19.18 \\
20 & 18.38 & 18.39 & 18.41 \\
22 & 17.94 & 17.95 & 17.96 \\
24 & 17.91 & 17.92 & 17.93 \\
26 & 18.08 & 18.09 & 18.10 \\
28 & 18.40 & 18.40 & 18.41 \\
30 & 18.79 & 18.80 & 18.94 \\
\hline
\end{tabular}

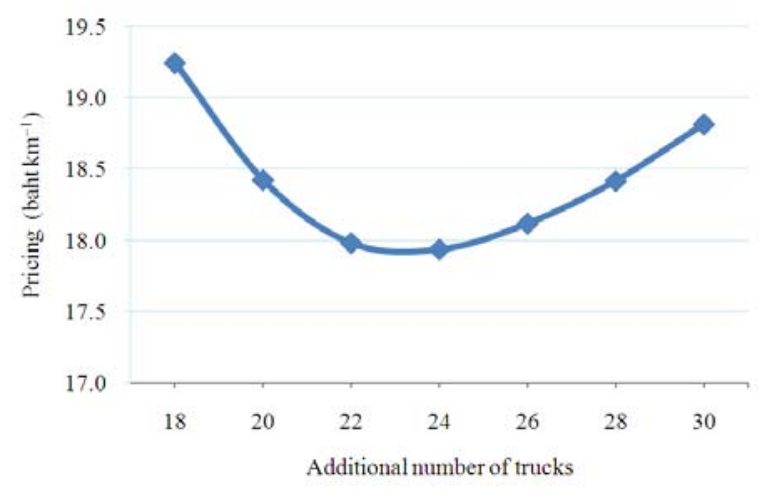

Fig. 4: The relationship between full truckload pricing with 95\% CVaR and amount of additional trucks

This will eventually lead to loss of customers. Taking advantage of the Conditional Value at Risk (CVaR) risk measurement technique, we can estimate the full truckload pricing depending on different levels of risk by changing different confident levels as demonstrated in Table 5. Therefore, transportation carriers will have room to negotiate with their customers while considering what an acceptable probability of loss is.

\section{DISCUSSION}

This study introduces a full truckload pricing model by applying a simulation model and the Conditional Value at Risk (VaR) technique to incorporate the effects of demand and service time uncertainties on truckload pricing. The developed methodology is tested using the actual operational data obtained from a truckload carrier operating in Bangkok, Thailand. A simulation model is developed to imitate the real-life daily operation of a full truckload network. The full truckload pricing proposed in this study is controlled by acceptable loss constraint. The simulation model can be used to determine the pricing at varying degrees of risk and can also be applied to investigate the effect of additional trucks on cost and price. To invest in new trucks, carriers need to trade off between the fixed cost of owning the trucks and the price of outsourcing. In addition, truck assignment rules that provide the appropriate assignment will simultaneously affect cost and price.

\section{CONCLUSION}

The numerical analysis full truckload pricing method in this study is suitable for transportation carriers who are risk averse. Transportation carriers in this group dislike risk and will stay away from high risk. However, if they stay extremely risk averse, pricing will be very high as a result. This will eventually lead to loss of customers. Taking advantage of the Conditional Value at Risk (CVaR) risk measurement technique, we can estimate the full truckload pricing depending on different levels of risk by changing different confident levels as demonstrated in Table 5. Therefore, transportation carriers will have room to negotiate with their customers while considering what an acceptable probability of loss is. Further studies may be conducted to apply Conditional Value at Risk (CVaR) to truckload pricing as well as to take into account other factors of uncertainties such as transit time uncertainty and so on. Moreover, full truckload pricing depends on each lane and less than truckload pricing will be considered.

\section{ACKNOWLEDGEMENT}

The researcher would like to express their sincere thanks to Faculty of Logistics and Transport, Burapha University for the financial support and transportation carriers who support all information required.

\section{REFERENCES}

Artzner, P., F. Delbaen, J.M. Eber and D. Heath, 1999. Coherent measures of risk. Math. Finance, 9: 203-228. DOI: 10.1111/1467-9965.00068

Embrechts, P., C. Kluppelberg and T. Mikosch, 1997. Modelling Extremal Events: For Insurance and Finance. 1st Edn., Springer, pp: 655. 
Rockafellar, R.T. and S. Uryasev, 2000. Optimization of conditional value at risk. J. Risk, 2: 21-41. http://www.ise.ufl.edu/uryasev/CVaR1_JOR.pdf

Saleh, A., Tsuji, T. and Oyama, T. 2009. Optimal bidding strategies for generation companies in a day-ahead electricity market with risk management taken into account. Am. J. Applied Sci., 2: 8-16. DOI: $10.3844 / .2009 .8 .16$

Suri, P.K. and R. Soni, 2006. Stochastically simulating low morale-a risk factor effect on risk management. J. Comput. Sci., 2: 370-372. DOI: 10.3844/jcssp.2006.370.372
Tapiero, C.S., 2005. Value at risk and inventory control. Eur. J. Operat. Res., 163: 769-775. DOI: 10.1016/j.ejor.2003.05.005

Uryasev, S., 2000. Conditional value-at-risk: optimization algorithms and applications. Financ. Eng. News, 14: 49-57. DOI: 10.1109/CIFER.2000.844598 